\title{
Strong thermospheric cooling during the 2009 major stratosphere warming
}

\author{
Huixin Liu, ${ }^{1,2}$ Eelco Doornbos, ${ }^{3}$ Mamoru Yamamoto, ${ }^{4}$ and S. Tulasi Ram ${ }^{4}$ \\ Received 23 April 2011; accepted 5 May 2011; published 21 June 2011.
}

[1] Thermospheric density simultaneously observed by the CHAMP and GRACE satellites in both the pre-dawn and afternoon local time sectors undergoes significant decrease across both hemispheres during the major stratospheric sudden warming (SSW) in January 2009. This decrease is largest in the equatorial region near the subsolar latitude, reaching $\sim 30 \%$ at $325 \mathrm{~km}$, and $45 \%$ at $475 \mathrm{~km}$ altitude in the afternoon sector. This large density drop demonstrates a substantial cooling of about 50 Kelvin in the equatorial upper thermosphere. Furthermore, the cooling varies clearly with longitude in terms of magnitude and the timing of the maximum cooling. Thermosphere cooling can have important impact on the ionosphere, as indicated by simultaneous plasma observations. Though many questions remain about what causes the cooling, our results open a new perspective for investigating the global coupling of the lower and upper atmosphere during SSWs. Citation: Liu, H., E. Doornbos, M. Yamamoto, and S. Tulasi Ram (2011), Strong thermospheric cooling during the 2009 major stratosphere warming, Geophys. Res. Lett., 38, L12102, doi:10.1029/2011GL047898.

\section{Introduction}

[2] Stratosphere sudden warming (SSW) is a meteorological event where the stratospheric temperature experiences a rapid and significant rise of more than a few tens Kelvin in the winter polar region. Its generation mechanism mainly involves planetary wave-mean wind interaction in the polar region and global-scale meridional circulation [Matsuno, 1971]. One particularly interesting aspect of SSW is that its effect is not confined to the polar stratosphere, but extends in altitude and latitude. In the polar region, mesosphere cooling and lower thermosphere warming have been predicted and observationally confirmed [see, e.g., Liu and Roble, 2002; Cho et al., 2004; Shepherd et al., 2010; Kurihara et al., 2010]. At middle latitudes, radar observations reveal alternating warming and cooling regions in the E- and F-region ionosphere [Goncharenko and Zhang, 2008]. At low and equatorial latitudes, significant cooling during SSWs has long been reported in the stratosphere [Fritz and Soules, 1972]. In the ionosphere at altitudes

\footnotetext{
${ }^{1}$ Department of Earth and Planetary Sciences, Kyushu University, Fukuoka, Japan.

${ }^{2}$ Space Environment Research Center, Kyushu University, Fukuoka, Japan.

${ }^{3}$ Aerospace Engineering, Delft University of Technology, Delft, Netherlands.

${ }^{4}$ Research Institute for Sustainable Humanosphere, Kyoto University, Uji, Japan.

Copyright 2011 by the American Geophysical Union. 0094-8276/11/2011GL047898
}

above $\sim 100 \mathrm{~km}$, a semi-diurnal perturbation has been identified during SSW events in various parameters like the vertical plasma drift and the total electron content (TEC) [Chau et al., 2009; Goncharenko et al., 2010].

[3] Since the daytime ion temperature closely follows that of the neutral temperature [Schunk and Nagy, 2000], the Millstone Hill radar observations shown by Goncharenko and Zhang [2008] suggest corresponding cooling in the upper thermosphere. This cooling would consequently cause thermospheric density decrease at a fixed altitude by reducing the scale height of the neutral atmosphere. To investigate if this were the case, we utilize thermospheric density observations from both CHAMP and GRACE satellites to study the well-reported 2009 major warming event.

\section{Data}

[4] Thermospheric density measurements from both the CHAMP and GRACE satellites are employed in this study. Both satellites were in near-circular polar orbits, sampling nearly simultaneously the afternoon (16-18 LT) and predawn (04-06 LT) sector during the SSW period between Jan. 15-30, 2009. The orbit height was about $325 \mathrm{~km}$ and $475 \mathrm{~km}$, for CHAMP and GRACE, respectively. During this period, the solar flux index of F10.7 was at a nearly constant level between 69-72 units. The Kp index was mostly below 2 , indicating an overall quiet geomagnetic condition with occasional minor disturbances on Jan. 19 and Jan. 26 (see Figure 1, bottom). For our study, all measurements are normalized to a fixed level of $F 10.7=70$ and $K p=1$, and to a fixed height of $325 \mathrm{~km}$ and $475 \mathrm{~km}$, for CHAMP and GRACE, respectively, The NRLMSISE00 and the IRI model 2007 version are utilized for the normalization. With this normalization, we minimize density variations due to solar and geomagnetic activities, and changes in satellites' orbit height. The method for deriving thermospheric density from accelerometer measurements are described by Liu et al. [2005] and Doornbos et al. [2010]. Due to large thermospheric wind in polar regions [Förster et al., 2008], density measurements below $\pm 60^{\circ}$ latitude are used in this study.

[5] Stratospheric temperature at $10 \mathrm{hPa}$ measured by the COSMIC satellite is utilized to describe the development of the 2009 major warming event. Compared to the frequently used NCEP reanalysis data, direct measurements from the COSMIC provide more accurate observations in polar regions as shown by, e.g., Kishore et al. [2009].

\section{Results}

\subsection{Strong Thermosphere Density Decrease}

[6] Figure 1 displays the longitudinally averaged thermosphere density during the SSW period between Jan. 15-30, 

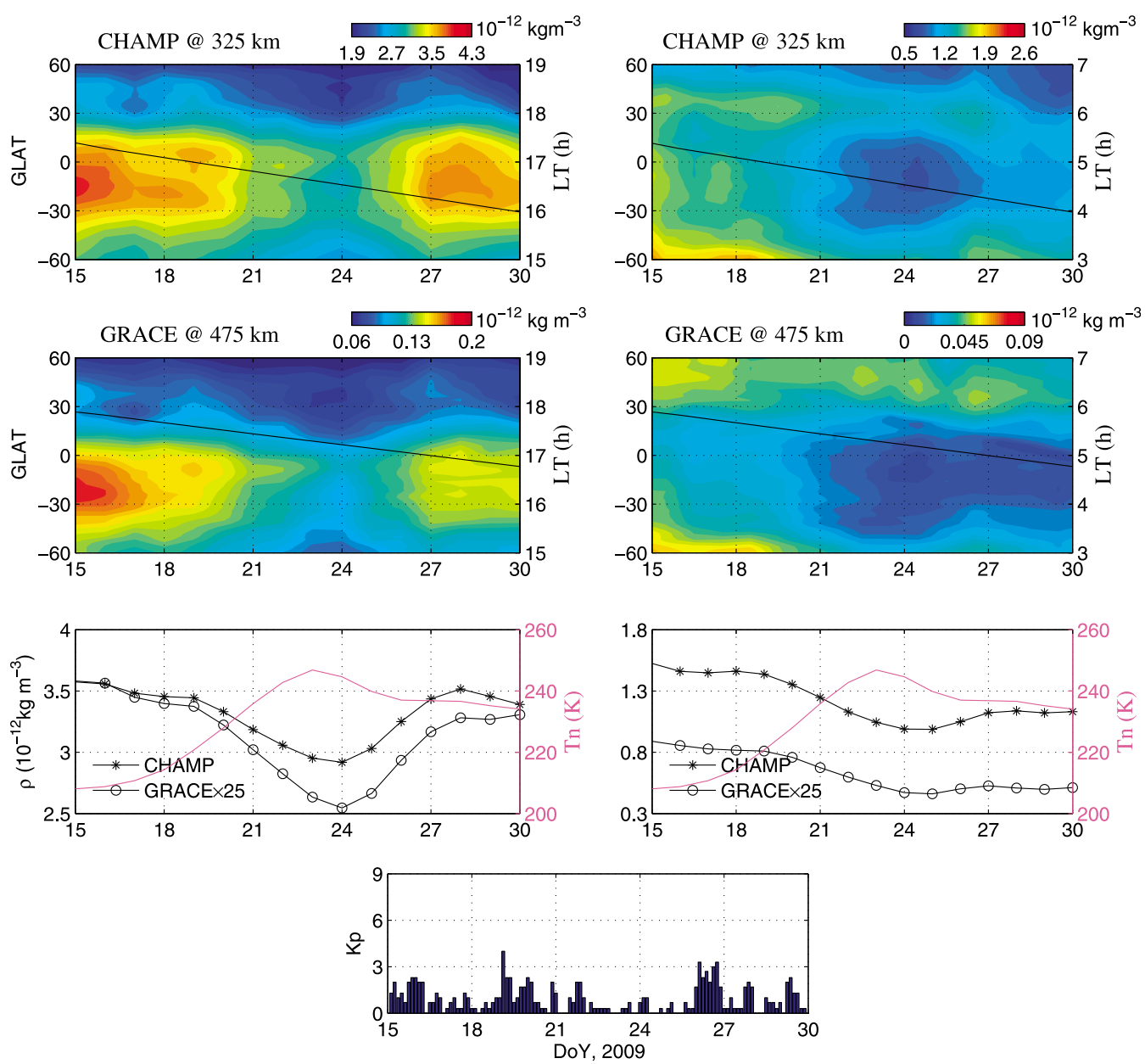

Figure 1. (top and middle top) Geographic latitude vs. day of year (DoY, 2009) distribution of the thermospheric mass density observed by CHAMP and GRACE, (left) for the afternoon sector (16-18 LT) and (right) for the pre-dawn sector (04-06 LT). Solid lines indicate the local time of the satellite tracks at corresponding days. (middle bottom) Equatorial density averaged within $30^{\circ} \mathrm{S}-30^{\circ} \mathrm{N}$. Note that the GRACE measurement is multiplied by 25 for easy display. The pink line is the stratospheric temperature at $10 \mathrm{hPa}$ from COSMIC observation, averaged over $70^{\circ} \mathrm{N}-90^{\circ} \mathrm{N}$, indicating the development of the SSW. A continuous decrease of the density is observed between Jan. 18-24, 2009. (bottom) Kp index during Jan. 15-30, 2009.

2009. It is immediately obvious that the thermospheric density observed by both satellites undergoes significant decrease, reaching a minimum on Jan. 24 , which is one day after the SSW peak. This phenomenon is observed in both the afternoon (Figure 1, left) and pre-dawn sectors (Figure 1, right), demonstrating its wide local time coverage. Since most features are similar in both sectors, due to limited space, we focus on the afternoon sector and examine more details below.

[7] Figure 2 shows more closely the development of the density decrease. Both satellites show consistent major features as the following. 1. Substantial density decrease occurs in two different regions already on Jan. 18, with one centering around the sub-solar latitude of $20^{\circ} \mathrm{S}$ and the other in the northern middle latitudes $\left(40^{\circ} \mathrm{N}\right) .2$. The density drop after Jan. 18 shows the largest depletion around sub-solar latitude, reaching $45 \%$ at $475 \mathrm{~km}$ and $30 \%$ at $325 \mathrm{~km}$ altitude on Jan. 24. 3. The southern hemisphere experiences a larger relative density decrease than the northern hemisphere. 4. The thermospheric density on Jan. 24 exhibits a prominent double-hump equatorial anomaly structure [ $\mathrm{Liu}$ et al., 2005] at both $325 \mathrm{~km}$ and $475 \mathrm{~km}$ altitudes. This may be an indication for enhanced upward propagating tides from the lower atmosphere, which contributes to the formation of the equatorial mass density anomaly [Miyoshi et al., 2011].

\subsection{Longitude Dependence}

[8] Since the equatorial region demonstrates the strongest disturbances as shown above, we focus on the equatorial region in the following. Figure 3 presents the average equatorial density in three different longitude sectors as the Peruvian, European and Indian sector. It is seen that temporal variations of the CHAMP and GRACE density agree with each other very well, hence lending further confidence in the observations. An obvious longitude dependence is observed in the density depletion in terms of magnitude and timing of the minimum. First, the magnitude of the depletion is largest in the Peruvian sector, where it reaches about $32 \%$, in comparison to $28 \%$ near $10^{\circ} \mathrm{E}$ and $20 \%$ near $75^{\circ} \mathrm{E}$ at $475 \mathrm{~km}$ altitude. Second, the timing of the minimum density varies. It occurs on Jan. 24 near $75^{\circ} \mathrm{W}$, but on Jan. 23 around $10^{\circ} \mathrm{E}$ and 

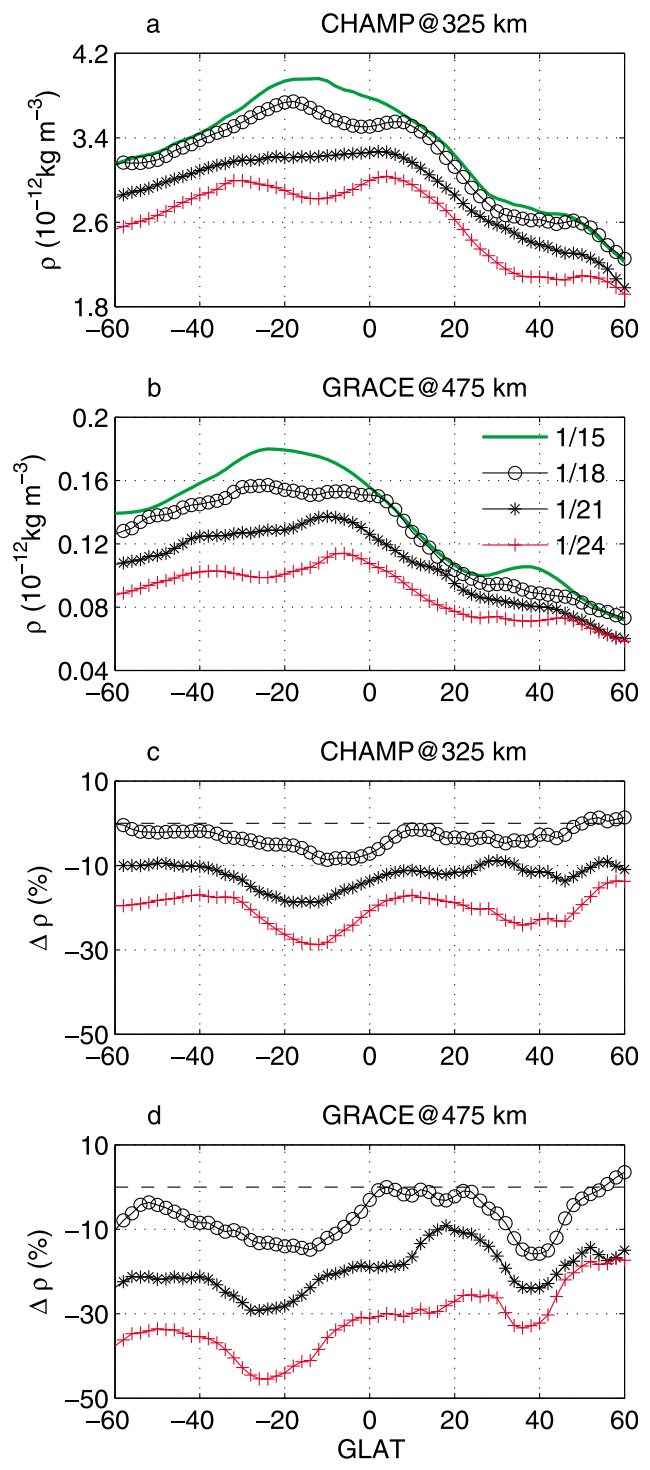

Figure 2. Latitudinal (geographic) profiles of the thermospheric density observed by CHAMP and GRACE on four representative days during the decreasing phase of the density in Figure 1. (a, b) The absolute density and (c, d) the relative percentage disturbance with respect to values on Jan. 15, 2009.

Jan. 25 at $75^{\circ} \mathrm{E}$. Finally, the relative density decrease is larger at GRACE height $(475 \mathrm{~km})$ than at CHAMP height $(325 \mathrm{~km})$ in all sectors. Strong longitudinal variation is also seen in the pre-dawn sector.

\section{Discussions}

[9] Both CHAMP and GRACE observations reveal pronounced neutral density reduction in the upper thermosphere during the 2009 major SSW event. The excellent agreement between these two satellites is a strong corroboration for the significance of this feature.

[10] Since thermospheric density is closely controlled by thermospheric temperature via scale height, this substantial reduction of the thermospheric density demonstrates significant cooling in the upper thermosphere. A first order estimation of the cooling can be obtained by combining the CHAMP and GRACE observations as explained below. Since neutral density decreases exponentially with altitude, we get $\rho_{G}=\rho_{C} e^{-\frac{\left(h_{G}-h_{C}\right)}{H_{n}}}$, where $h_{G}=475 \mathrm{~km}$ and $h_{C}=$ $325 \mathrm{~km} . H_{n}=\frac{k T_{n}}{\bar{m} g}$ is the average thermospheric scale height, with $k$ being the Boltzmann constant, $g$ the gravitational acceleration, and $\bar{m}$ the mean mass of neutral particles. Hereby, we have assumed that the neutral temperature $\left(T_{n}\right)$ and the mean mass between $325-475 \mathrm{~km}$ altitude does not vary much, which is justified by the NRLMSISE00 model. Taking $\bar{m}=16.4 m_{p}$ from the NRLMSISE00 model, where $m_{p}$ is the proton mass, and density measurements of $\rho_{G}$ and $\rho_{C}$ from GRACE and CHAMP satellites, a straightforward estimation of $T_{n}$ can be obtained.

[11] The estimated $T_{n}$ for the Peruvian sector is shown in Figure 4. It shows that during the 2009 SSW, the neutral temperature in the upper thermosphere has dropped about 50 Kelvin. Alternatively, we have run the NRLMSISE00 model to estimate the temperature drop by fitting its thermospheric density output to the observed values from CHAMP/GRACE. This yields a temperature drop about $40 / 60$ Kelvin, hence further corroborating the analytical estimation method used above. Since F-region ion temperature closely follows that of the neutral temperature during day [Schunk and Nagy, 2000], the thermosphere
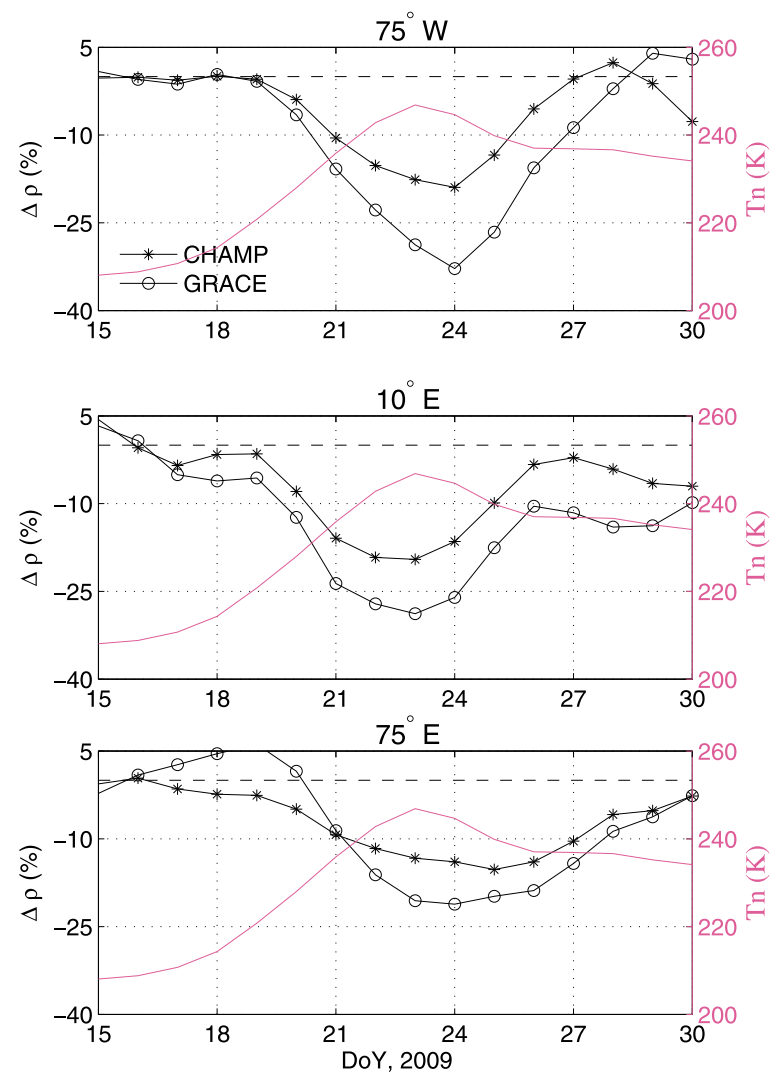

Figure 3. Disturbance (in percentage) of the thermospheric density averaged within $30^{\circ} \mathrm{S}-30^{\circ} \mathrm{N}$ for the Peruvian sector around $75^{\circ} \mathrm{W}$, the European sector around $10^{\circ} \mathrm{E}$, and the Indian sector around $70^{\circ} \mathrm{E}$. Pink lines are same as that shown in Figure 1. The average values between Jan. 1517,2009 is taken as the quiet reference. 


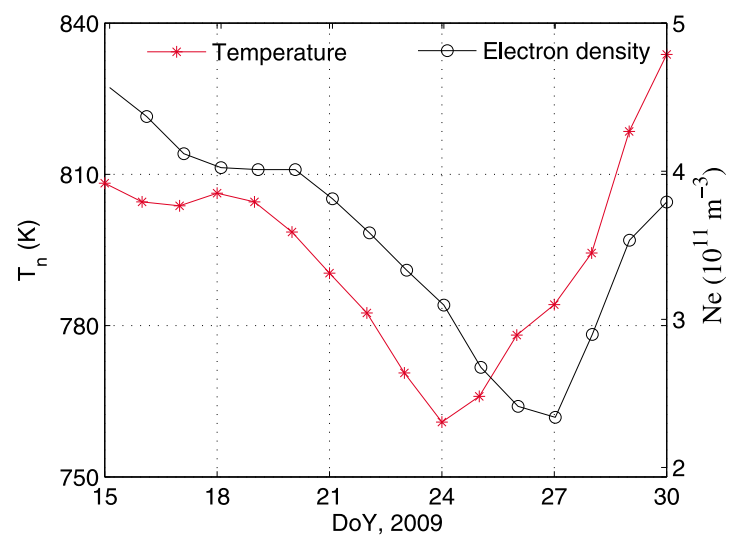

Figure 4. Thermospheric temperature estimated from the CHAMP and GRACE density observations shown in the top panel of Figure 3, along with the corresponding plasma density from CHAMP also averaged between $30^{\circ} \mathrm{S}-$ $30^{\circ} \mathrm{N}$. A drop about $50 \mathrm{~K}$ is seen in $T_{n}$ in the equatorial region during the $2009 \mathrm{SSW}$.

cooling would lead to similar decrease of the ion temperature. Observations of F-region ion temperature decrease of 40 Kelvin has been reported by Goncharenko and Zhang [2008] during a different SSW event, which is similar to the neutral temperature drop obtained here.

[12] Thermosphere cooling can affect the ionosphere in two obvious ways. First, it reduces the scale height of the neutrals, hence changing the neutral density and composition at a fixed altitude. This would affect the plasma density directly via photoionization and chemical recombination processes. Second, it leads to the ion temperature drop, hence a reduced plasma scale height. This causes plasma density decrease at altitudes near and above the F2 peak region. Observations of plasma density at $325 \mathrm{~km}$ altitude from CHAMP during the 2009 SSW exhibits a significant depletion, which closely follows the thermospheric temperature with a delay of 1-2 days (see Figure 4). Although the major cause of this equatorial plasma depletion is likely the strong downward plasma drift as pointed out by Chau et al. [2009] and Goncharenko et al. [2010], features in Figure 4 may indicate additional contribution from the thermospheric cooling via effects explained above. Furthermore, the longitudinal variation with the strongest thermospheric cooling in the Peruvian sector also finds good agreement with the simulation results of Liu et al. [2010], showing strongest ionospheric response in this sector.

[13] Considering the extremely quiet geomagnetic activity level $(K p<2)$ during Jan. 19-25, geomagnetic activity effect can almost be confidently excluded. Thus, this thermospheric cooling may be potentially related to the occurrence of the SSW event. The physical mechanism leading to the cooling is an interesting question. Cooling in the tropical stratosphere during SSW events is well known and explained as a result of upward air motion in the tropics related to the meridional circulation during SSWs [Fritz and Soules, 1972]. On the other hand, cooling in the ionosphere at middle latitudes has also been observed [Goncharenko and Zhang, 2008]. In view of these results, two routes could be speculated for the cause of the cooling in the upper ther- mosphere. First, the SSW perturbs the upper thermosphere in the polar region, whose effect subsequently propagates to middle and low latitudes within the upper regime of the thermosphere via global circulation or atmospheric waves. This route is in analog to the disturbance propagation during magnetic storms, though the nature of the two phenomena and the propagation time are completely different. The other could be that the SSW first causes a perturbation in the equatorial stratosphere, then propagates upward to affect the equatorial upper atmosphere. This route is in analog to the coupling processes related to the wave- 4 phenomena [Liu et al., 2009]. Our observations seem to support both routes operating simultaneously, as two separate cooling regions are clearly identified (see Figure 1). It is highly interesting to examine the plausibility of this speculation with numerical models.

[14] To conclude, our analysis of observations from two satellites reveals, for the first time, strong cooling in the upper thermosphere during stratospheric sudden warming events. This cooling shows clear longitudinal dependence and can have significant effect on the plasma. Though many questions remain about what causes the cooling, our results open a new perspective for investigating thermosphere-ionosphere response to SSWs in particular, and for global coupling of the lower and upper atmosphere in general.

[15] Acknowledgments. We thank Y. Kakinami and M. Kamogawa and J. J. Berthelier for helpful discussions and C. Stolle for providing the CHAMP plasma density data.

[16] The Editor thanks Gordon Shepherd and an anonymous reviewer for their assistance in evaluating this paper.

\section{References}

Chau, J. L., B. G. Fejer, and L. P. Goncharenko (2009), Quiet variability of equatorial $\mathrm{E} \times \mathrm{B}$ drifts during a sudden stratospheric warming event, Geophys. Res. Lett., 36, L05101, doi:10.1029/2008GL036785.

Cho, Y.-M., G. G. Shepherd, Y.-I. Won, S. Sargoytchev, S. Brown, and B. Solheim (2004), MLT cooling during stratospheric warming events, Geophys. Res. Lett., 31, L10104, doi:10.1029/2004GL019552.

Doornbos, E., J. van den IJssel, H. Lühr, M. Förster, and G. Koppenwallner (2010), Neutral density and crosswind determination from arbitrarily oriented multiaxis accelerometers on Satellites, J. Spacecr. Rockets, 47, $580-589$.

Förster, C., S. Rentz, W. Köhler, H. Liu, and S. E. Haaland (2008), IMF dependence of high-latitude thermospheric wind pattern derived from CHAMP cross-track measurements, Ann. Geophys., 26, 1581-1595.

Fritz, S., and S. D. Soules (1972), Planetary variations of stratospheric temperature, Mon. Weather Rev., 100, 582-589.

Goncharenko, L., and S.-R. Zhang (2008), Ionospheric signatures of sudden stratospheric warming: Ion temperature at middle latitude, Geophys. Res. Lett., 35, L21103, doi:10.1029/2008GL035684.

Goncharenko, L. P., J. L. Chau, H.-L. Liu, and A. J. Coster (2010), Unexpected connections between the stratosphere and ionosphere, Geophys. Res. Lett., 37, L10101, doi:10.1029/2010GL043125.

Kishore, P., S. P. Namboothiri, J. H. Jiang, V. Sivakumar, and K. Igarashi (2009), Global temperature estimates in the troposphere and stratosphere: A validation study of COSMIC/FORMOSAT-3 measurements, Atmos. Chem. Phys., 9, 897-908.

Kurihara, J., Y. Ogawa, S. Oyama, S. Nozawa, M. Tsutsumi, C. M. Hall, Y. Tomikawa, and R. Fujii (2010), Links between a stratospheric sudden warming and thermal structures and dynamics in the high-latitude mesosphere, lower thermosphere, and ionosphere, Geophys. Res. Lett., 37, L13806, doi:10.1029/2010GL043643.

Liu, H., H. Lühr, V. Henize, and W. Köhler (2005), Global distribution of the thermospheric total mass density derived from CHAMP, J. Geophys. Res., 110, A04301, doi:10.1029/2004JA010741.

Liu, H., M. Yamamoto, and H. Lühr (2009), Wave-4 pattern of the equatorial mass density anomaly: A thermospheric signature of tropical deep convection, Geophys. Res. Lett., 36, L18104, doi:10.1029/ 2009GL039865. 
Liu, H.-L., and R. G. Roble (2002), A study of a self-generated stratospheric sudden warming and its mesospheric-lower thermospheric impacts using the coupled TIME-GCM/CCM3, J. Geophys. Res., 107(D23), 4695, doi:10.1029/2001JD001533.

Liu, H.-L., W. Wang, A. D. Richmond, and R. G. Roble (2010), Ionospheric variability due to planetary waves and tides for solar minimum conditions, J. Geophys. Res., 115, A00G01, doi:10.1029/2009JA015188.

Matsuno, T. (1971), A dynamical model of the stratospheric sudden warming, J. Atmos. Sci., 28, 1479-1494.

Miyoshi, Y., H. Fujiwara, H. Jin, H. Shinagawa, H. Liu, and K. Terada (2011), Model study on the formation of the equatorial mass density anomaly, J. Geophys. Res., doi:10.1029/2010JA016315, in press.

Schunk, R. W., and A. F. Nagy (2000), Ionospheres: Physics, Plasma Physics, and Chemistry, Cambridge Univ. Press, Cambridge, U. K.
Shepherd, G. G., Y.-M. Cho, and M. G. Shepherd (2010), Mesospheric temperature observations at Resolute $\left(75^{\circ} \mathrm{N}\right)$ in the context of solar flux and quasi-biennial variations, J. Geophys. Res., 115, A08301, doi:10.1029/2009JA015126.

E. Doornbos, Aerospace Engineering, Delft University of Technology, Kluyverweg 1, NL-2629 HS Delft, Netherlands.

H. Liu, Space Environment Research Center, Kyushu University, Fukuoka 812-8581, Japan. (huixin@serc.kyushu-u.ac.jp)

S. Tulasi Ram and M. Yamamoto, Research Institute for Sustainable Humanosphere, Kyoto University, Gokasho, Uji, Kyoto 611-0011, Japan. 